\title{
Territorialisation à distance par caméra vidéo : Perception de la vidéosurveillance au quartier des Pâquils à Genève
}

\section{Francisco Klauser and Raoul Kaenzig}

Institut de Géographie, Université de Neuchâtel, Espace Louis Agassiz 1, 2000 Neuchâtel, Switzerland

Correspondence to: Francisco Klauser (francisco.klauser@unine.ch)

and Raoul Kaenzig (raoul.kaenzig@unine.ch)

Received: 8 February 2017 - Revised: 25 April 2017 - Accepted: 1 May 2017 - Published: 6 July 2017

\begin{abstract}
Résumé. Drawing upon two public opinion surveys conducted in Geneva in 2015 and 2016, the paper explores how video surveillance was lived and perceived by the residents of the monitored Pâquis area, as well as by the population at large. This study shows that the delegation of control to systems and individuals that are both spatially and socially detached from the monitored areas also results in a kind of 'mental distance' that characterizes the relation between the monitored individuals and the system: People quickly forget about the cameras and withdraw from any conscious attempt to imagine what might be going on behind their back. The system thus gradually loses its relevance in people's everyday life. This, in turn, limits the symbolic power of the cameras to enduringly revitalize, and thus to properly re-territorialize, the monitored areas.
\end{abstract}

\section{Introduction}

La vidéosurveillance de l'espace public connaît un essor remarquable depuis les années 1990. En France, le chiffre d'affaire du secteur est passé de 360 millions d'euros à 910 millions d'euros entre 1996 et 2008. En Angleterre, on estime que plus de cinq millions de caméras sont opérationnelles (Heilmann et al., 2012). En Suisse aussi, les systèmes de vidéosurveillance sont désormais un outil sécuritaire de plus en plus répandu (Guéniat et Klauser, 2013). Tous les aéroports, la plupart des gares, les systèmes de transports publics ainsi que les grands axes routiers en sont équipés. De plus en plus de communes autorisent la pose de caméras dans les préaux des établissements scolaires (Taylor, 2013). Les gardes-frontières prévoient de munir les postes-frontières de centaines de caméras. Enfin, les espaces publics en milieu urbain ne sont pas en reste. Pionnière, la ville d'Olten a installé un tel dispositif dans les années 2000 déjà (Klauser, 2006).

A l'heure actuelle, de nombreux nouveaux projets voient le jour, dont celui du quartier des Pâquis à Genève. Ce système comprend vingt-neuf caméras installées durant l'automne 2014 dans un périmètre compris entre la place de Cornavin et les rues du Mont-Blanc, de Berne, Pellegrino-Rossi,
de-Monthoux et de Fribourg (cf. "zone pilote", Fig. 1). Il s'agit du dispositif de caméras le plus étendu (spatialement) et avancé (techniquement) de Suisse, focalisé sur des espaces publics extérieurs avec visionnage des images en temps réel. L'investissement lié à son installation s'est élevé à 1.1 millions de francs (Département de la sécurité et de l'économie du Canton de Genève, 2014) et s'inscrit dans le cadre de la mise en œuvre de la convention de lutte contre la criminalité, adoptée conjointement par le Ministère public et le Conseil d'Etat le 29 août 2012 et rééditée le 17 septembre 2014 (République et Canton de Genève, 2014). Les caméras sont pilotées depuis une centrale de visionnage installée dans les locaux de la police cantonale, au Nouvel hôtel de police. Une dizaine de collaborateurs se relaient 20 sur $24 \mathrm{~h}$ et 365 jours par an pour visionner les images et diriger les caméras.

En s'appuyant sur deux enquêtes par questionnaire, conduites en 2015 et en 2016, cet article s'intéresse à la manière dont ce dispositif de caméras est vécu et perçu par les habitants du quartier des Pâquis ainsi que par la population genevoise plus généralement ${ }^{1}$. Cette analyse sera complétée

\footnotetext{
${ }^{1}$ Cette étude a été mandatée par le Département de la Sécurité et de l'Economie du Canton de Genève. Elle a été conduite de manière
} 
par un deuxième article rédigé par les mêmes auteurs, qui adoptera une perspective analytique et méthodologique complémentaire, focalisée sur les effets préventifs du même projet de vidéosurveillance et sur l'usage des caméras en matière d'opérations policières et d'élucidations de crimes. Les deux articles poursuivent une approche géographique pour étudier la vidéosurveillance, visant à mieux comprendre l'efficacité des caméras comme instrument de territorialisation symbolique et informationnelle des espaces observés à distance. Ci-dessous, nous situons cette approche au sein de la littérature thématique existante, avant de passer aux explications méthodologiques et à l'analyse.

\section{La vidéosurveillance comme outil de territorialisation à distance}

La vidéosurveillance offre un regard techno-médiatisé sur l'espace. Elle présente ainsi un rapport direct aux lieux surveillés, permettant d'en extraire des informations de par sa capacité de transfert et d'enregistrement de données visuelles et d'y projeter des informations de par son pouvoir symbolique. Dans les deux cas, exprimant des intentions focalisées sur des espaces filmés, elle fonctionne comme un outil de territorialisation au sens raffestinien du terme (Raffestin, 1980), dont l'articulation spatiale reflète les représentations et objectifs spécifiques de ses usagers (Zurawski, 2014). Par "territorialisation", nous entendons, en suivant Raffestin, le processus par lequel un sujet individuel ou collectif établit une relation avec un espace, à travers des médiateurs concrets (outils, pratiques) et/ou abstraits (connaissances, représentations) (Raffestin, 1980:130). Comme "objet de visées intentionnelles et d'actions" (Raffestin, 1980:137), l'espace se transforme ainsi en territoire.

Dans le cas de la vidéosurveillance, les caméras peuvent notamment viser des points particuliers dans l'espace (lorsque des endroits spécifiques sont monitorés de manière uniforme et fixe), observer des lignes de séparation ou de connexion (lorsque les caméras sont positionnées verticalement, par exemple pour la surveillance des frontières ou des infrastructures de transport), ou encore couvrir des surfaces plus larges (la surveillance d'espaces plus étendus grâce à des caméras pivotantes par exemple). La disposition et la logique spatiale de la vidéosurveillance produisent ainsi une hiérarchisation spatiale en des zones plus ou moins investies par son pouvoir informationnel et symbolique (Klauser, 2001).

Séparant les "espaces de contrôle" des "espaces contrôlés", cette surveillance spatiale s'effectue, fondamentalement, à distance (Akrich et Méadel, 1999). À cette distance spatiale s'ajoute une distanciation sociale, qui découle des nouveaux acteurs qui interviennent à travers la vidéosur-

indépendante entre novembre 2014 et novembre 2016 et suivie par un groupe d'accompagnement composé de représentants-es de la police, de la ville, des habitants-es du quartier et des travailleurs-ses du sexe (Kaenzig et Klauser, 2016).

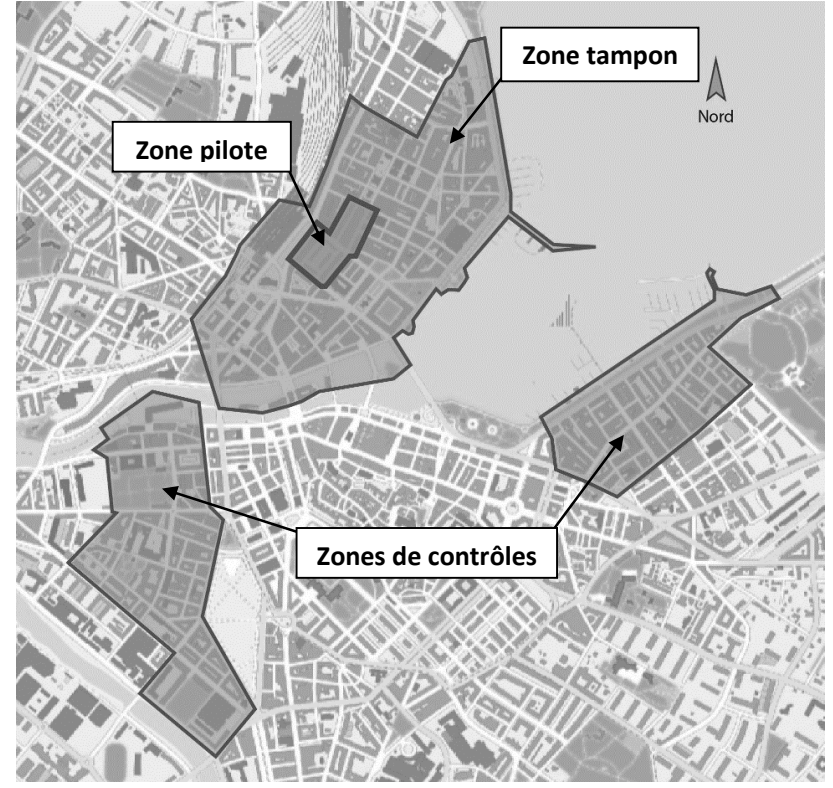

Figure 1. Carte des périmètres retenus pour l'enquête par questionnaire. Source : SAS Police cantonale de Genève et Institut de géographie UniNE, 2015.

veillance dans la relation entre le surveillant et le surveillé (Marx, 1991; Ruegg et al., 2006). Par exemple, les fabricants de systèmes de caméras, les fournisseurs de logiciels, ou encore les opérateurs qui visionnent et manœuvrent les caméras sont autant de nouveaux intermédiaires agissant dans le domaine de la sécurité publique. Si le lien entre les citoyens et les autorités en charge de la sécurité n'est pas rompu, il se trouve rallongé du fait qu'il se fait désormais par l'entremise de ces différents acteurs (Ruegg et al., 2007).

La surveillance techno-médiatisée par caméra vidéo se distingue ainsi des mécanismes de contrôle social qui caractérisent traditionnellement l'espace public. Comme espace à usage public et à accessibilité généralisée (Barbichon, 1990; Jeudy, 1990), l'espace public rassemble et met en scène des personnes qui ne se connaissent pas a priori, mais qui occupent le même lieu au même moment. La régulation de ces espaces peut alors être exercée par toutes les personnes simultanément présentes (Koresec-Serfaty, 1990; Pellegrino et al., 1990). Par contraste, la télé-surveillance par caméra vidéo implique un tout autre type de régulation et de rapport entre régulateur et régulé. Le monde derrière les caméras n'est pas accessible aux usagers des espaces surveillés. La logique de visibilité réciproque des relations sujet - sujet au sein de l'espace public est remplacée par une logique relationnelle unilatérale sujet - objet, ou encore par une relation institution - individu (Ruegg et al., 2007). L'individu qui entre dans le champ de vision des caméras n'est plus sujet de communication mais devient simple objet d'information, sans avoir lui-même connaissance de l'observateur. En découle un "pouvoir à distance", i.e. une capacité d'action en 
retrait, fondamentalement opaque et difficilement questionnable.

Or, la mise à distance spatiale et sociale du type de régulation induit par la vidéosurveillance ne soulève pas seulement des questions du point de vue du pouvoir et de la transparence du dispositif, mais affecte également l'efficacité des caméras comme outils de territorialisation des espaces filmés. Ci-dessous, nous développons cet argument plus amplement en trois axes, relatifs à (1) l'utilité de la vidéosurveillance à des fins opérationnelles, (2) l'efficacité préventive des caméras, et (3) les effets des caméras sur le sentiment de sécurité des usagers des espaces surveillés. Dans le premier cas, le pouvoir de territorialisation réside dans la capacité des caméras à transmettre des informations afin de gérer des zones surveillées. Pour les deux autres, il s'agit de la capacité de la caméra, à travers son pouvoir symbolique, de territorialiser des espaces en affectant leurs qualités sémantiques, qui s'expriment respectivement en termes de sécurité objective et subjective. Les deux dimensions principales, pratiques et sémantiques, du processus de territorialisation selon Raffestin (Raffestin, 1980) sont ainsi touchées par les caméras vidéo.

Sur la base de cette discussion initiale, l'analyse développée dans le présent article se concentre sur le troisième axe, "perception des caméras et sentiment de sécurité", afin d'apporter suffisamment de profondeur et de différenciation à notre propos. Rappelons néanmoins que dans un deuxième article, nous aborderons également les deux autres axes, liés aux usages opérationnels et aux effets préventifs des caméras.

\subsection{Territorialisation à distance et effets opérationnels de la vidéosurveillance}

Une première manière d'étudier la territorialisation à distance par caméra vidéo réside dans l'analyse de leur utilité pour des opérations policières (Norris et Armstrong, 1998; November et al., 2003; Smith, 2015). Le pouvoir de territorialisation des caméras est ici associé à leur capacité de transmettre des informations visuelles, qui contribuent à la gestion en temps réel des zones filmées (le flagrant délit, le télé-pilotage d'opérations policières, etc.). Si la littérature existante souligne une variété de facteurs qui affectent cet usage de la vidéosurveillance, allant de la qualité des collaborations entre opérateurs et patrouilles jusqu'aux dimensions techniques et aux emplacements des caméras (Home Office, 2007), nous nous concentrons ici sur deux facteurs principaux, liés directement à la problématique de la distance induite par la vidéosurveillance.

Premièrement, l'usage des caméras à des fins opérationnelles soulève des questions importantes concernant les possibilités et les problèmes d'intelligibilité de la réalité observée à distance. La vidéosurveillance procède à un cadrage des portions du territoire prises en image, sorties de leur contexte d'origine et réduites par rapport à la complexité originelle des situations filmées, pour ensuite être re- contextualisées dans une situation de visionnage et de traitement d'images (Ruegg et al., 2006). Dans ce processus, la caméra n'offre qu'une seule perspective, souvent surélevée, et rend intelligible à sa façon uniquement, une portion d'espace. À son regard échappe une multitude d'éléments tels que les ambiances, les sons, les odeurs, etc. Par conséquent, si les caméras permettent de voir le déroulement d'une altercation, il est souvent difficile de comprendre les raisons et les motivations des protagonistes (Norris et Armstrong, 1998; Armstrong et Giulianotti, 1998). Comme l'un des avocats impliqués dans une affaire d'agression aux Pâquis l'a soulevé dans la presse : "Les images ne disent pas grandchose sur les causes du conflit [...]. Qui provoque? Qui insulte? Il n'y a pas de son" (Mendecino, 2015). Souvent, ce problème est souligné par les opérateurs eux-mêmes, qui regrettent le manque de contact multi-sensoriel avec les zones filmées (Klauser, 2007).

Le deuxième "problème de distance", limitant l'efficacité opérationnelle des caméras, concerne l'absence d'interactions avec les usagers des espaces filmés. Aux Pâquis, le personnel surveillant peut "marquer" des événements suspects sur les enregistrements, mais n'a aucune possibilité de communiquer avec les personnes surveillées. Pour faire face à ce type de limites, certaines agglomérations en Angleterre et en France se sont dotées de caméras équipées de haut-parleurs. Censés permettre aux opérateurs de s'adresser oralement aux personnes filmées, ces systèmes connaissent pourtant des problèmes d'audibilité liés au bruit de l'environnement urbain (Mach et Feuz, 2007).

\subsection{Territorialisation à distance et effets préventifs de la vidéosurveillance}

Les recherches relatives aux effets de la vidéosurveillance en matière de prévention de la criminalité offrent une deuxième manière d'étudier la territorialisation à distance par caméra vidéo. Il s'agit ici d'investiguer dans quelle mesure les caméras "informent" véritablement des espaces filmés, en y prévenant des actes délictueux (Ditton et Short, 1999; Fyfe et Bannister, 1998; Williams et al., 2000; Sivarajasingam et al., 2003; Fonteneau, 2008; Zehnder, 2011). La littérature existante met en avant trois grands facteurs qui limitent l'efficacité de la vidéosurveillance à ce niveau.

Premièrement, il apparait que l'efficacité préventive de la vidéosurveillance dépend du type de criminalité analysé. Par exemple, l'agentivité symbolique de la vidéosurveillance s'avère plus efficace dans le cas de la criminalité rationnelle (qui intègre une réflexion coûts-bénéfices de la part des délinquants) que dans le cas de la criminalité affective (émotionnelle ou impulsive, par exemple sous l'effet de l'alcool ou de drogues) (Brown, 1995). Deuxièmement, si l'installation de caméras peut - pour certains types de délits et dans un premier temps - contribuer à améliorer la sécurité d'un espace, les effets préventifs sur le long terme s'avèrent moins concluants (Welsh et Farmington, 2002). Les délin- 
quants s'habituent à la présence de caméras et finissent par les ignorer ou même les oublier (Ditton, 2000; Armitage, 2002; Gill et Spriggs, 2005; Klauser, 2007). Le présent article s'intéresse tout particulièrement à ce phénomène d'oubli et d'indifférence vis-à-vis des caméras du point de vue de la population dans son ensemble. Troisièmement, l'efficacité de la vidéosurveillance n'est pas seulement limitée dans le temps, mais aussi dans l'espace et peut avoir pour effet de déplacer la criminalité dans des zones dépourvues de caméras (Tilley, 1998; Skinns, 1998; Bowers et Johnson, 2003; Carli, 2009; Waples et al., 2009). En somme, si le caractère spatialement distant de la vidéosurveillance permet de surveiller plusieurs espaces de manière simultanée (selon le nombre de caméras), flexible (caméras pivotantes) et à des échelles variables (zoom), les résultats en matière de prévention de la criminalité restent souvent limités.

\subsection{Territorialisation à distance et sentiment de sécurité}

Une troisième manière d'aborder, et de relativiser, le pouvoir de territorialisation à distance par caméra vidéo réside dans l'étude de la perception de la vidéosurveillance et de ses effets par les usagers des espaces filmés. Cet outil permet-il véritablement de changer les qualités des espaces surveillés, tels qu'ils sont vécus et perçus?

La littérature scientifique démontre que la population adopte une attitude plutôt pragmatique vis-à-vis de la vidéosurveillance; on accepte les caméras parce qu'on estime ne rien avoir à cacher (Honess et Charman, 1992). Cette opinion relève cependant plutôt d'un consentement tacite que d'une revendication explicite. La population préfère une présence renforcée d'agents de police aux caméras, si elle pouvait librement choisir (Klauser, 2006). Sur cette base, force est de constater que des mesures qui favorisent la proximité sont préférées à celles qui créent de la distance, et ceci notamment pour la surveillance d'espaces considérés comme personnels, voire intimes. Ainsi, la population accepte aisément l'utilisation de la vidéosurveillance dans des parkings et pour des passages piétons, mais se montre plus critique face aux caméras qui se trouvent dans les parcs publics ou encore dans des quartiers d'habitation (Ditton, 2000:700; Koskela, 1999).

Un autre enseignement important provenant des enquêtes sur la perception de la vidéosurveillance est que son efficacité du point de vue du sentiment de sécurité "dépend autant (sinon plus) de la capacité des promoteurs des systèmes à mobiliser la population autour de leur projet que des moyens techniques et humains mis en œuvre" (Heilmann, 2003:101). Il paraît ainsi que plus les habitants sont informés de la présence, des objectifs et de l'utilisation des caméras - en d'autres termes, plus ils arrivent à s'approprier cette technologie et à surmonter ainsi la mise à distance spatiale et sociale qu'elle implique - plus ils se sentent concernés par le projet et donc potentiellement rassurés (Ditton, 1998). Dans notre analyse ci-dessous, nous approfondissons la discussion de ce point, en étudiant la perception populaire d'un dispositif de caméras particulièrement médiatisé, en raison du caractère pionnier du projet et de sa réalisation dans un quartier de prostitution et de trafic de drogue.

\subsection{La distance mentale}

Pris ensemble, les trois approches de la territorialisation à distance par caméra vidéo soulignent qu'en déléguant la régulation d'un espace à des systèmes et individus qui en sont séparés spatialement et socialement, la vidéosurveillance crée aussi une certaine distance mentale, qui s'exprime, pour les surveillés, sous la forme d'un oubli et d'un désintérêt vis-à-vis des caméras, ainsi que d'une incapacité à véritablement saisir les objectifs derrière le dispositif et les usages qui en sont faits. Cette distance mentale découle directement de la distance spatiale et sociale engendrée par la vidéosurveillance (Wünsch, 2001; Klauser, 2007). Comme il n'y a pas d'interactions possibles avec le dispositif et qu'il est peu visible, son impact n'est pas immédiatement perceptible et les personnes qui le manipulent ne sont pas connues du public (Klocke, 2001). Puisque les caméras sont éloignées, spatialement et socialement parlant, elles finissent par devenir une abstraction à laquelle on ne fait plus attention.

Notre analyse de la perception populaire des caméras aux Pâquis approfondit encore cette réflexion. Nous étudions dans un premier temps les connaissances populaires vis-à-vis des caméras ainsi que l'acceptabilité sociale de celles-ci, en postulant que pour territorialiser un espace du point de vue de son vécu et de sa perception, il est nécessaire que les caméras soient connues et jugées utiles par la population. Deuxièmement, nous investiguons la manière dont la population évalue sa propre sécurité avant et après l'installation des caméras. Finalement, nous abordons les effets de la vidéosurveillance sur l'image et la fréquentation du quartier.

\section{Approche méthodologique}

Notre analyse s'appuie sur deux enquêtes quantitatives par questionnaire, réalisées en juin 2015 (1151 répondants sur 6000 questionnaires administrés, taux de réponse de $20.5 \%$ ) et en mai 2016 (1245 répondants sur 6000 questionnaires administrés, taux de réponse de $22 \%$ ). Pour les deux enquêtes la méthode d'échantillonnage a été identique afin de permettre le suivi et la comparaison de la perception populaire de la vidéosurveillance aux Pâquis et de ses effets à travers le temps. Pour chaque enquête, sur la base des identités fournies par l'Office cantonal de la population et des migrations de Genève, un échantillon respectant la structure par âge et par sexe du canton a été sélectionné pour quatre périmètres géographiques :

- Zone dotée de caméras (zone pilote) : 1564 personnes sélectionnées en 2015 et 1631 personnes en 2016. Pour cette zone, la totalité des habitants a été sélectionnée pour les deux enquêtes, sa taille restreinte et le nombre 


\section{Connaissances du dispositif de caméras en 2015}
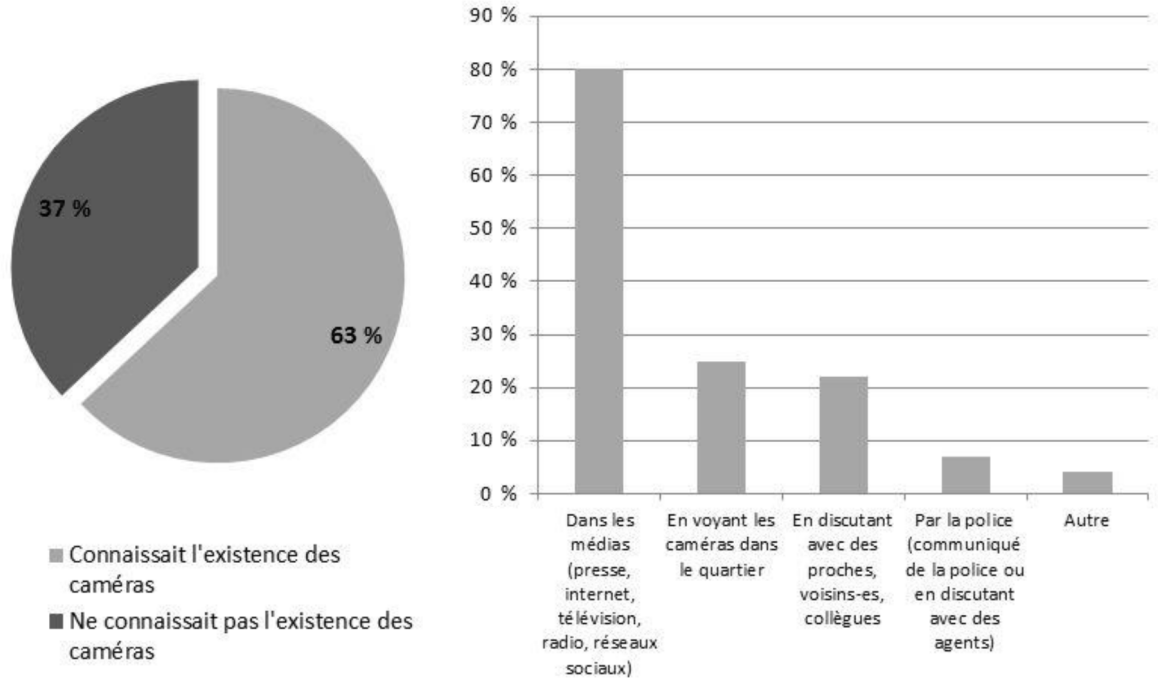

Figure 2. Connaissances des répondants des zones pilote, tampon et de contrôle quant à l'existence du dispositif de caméras et leurs sources (Source : questionnaire 2015, $N=958$ ).

limité d'habitants ne permettant pas de procéder à un échantillonnage.

- Zone limitrophe du périmètre surveillé (zone tampon) : 1500 personnes sélectionnées en 2015 et en 2016.

- Deux zones de contrôle avec des caractéristiques similaires aux Pâquis, en matière de structure démographique et de taux de criminalité, mais qui ne sont pas équipées de caméras (Coulouvrenière-Rois et EauxVives) : $1500+1500$ personnes sélectionnées en 2015 et en 2016. La comparaison avec ces quartiers permet de mieux mettre en évidence le "facteur camerá" pour la zone pilote.

Les deux enquêtes ont été réalisées à l'aide du logiciel de sondage en ligne Qualtrics. Les habitants des zones sélectionnées ont été contactés par voie postale avec une lettre signée par la cheffe de la Police genevoise les invitant à se rendre sur une page internet hébergée par l'Université de Neuchâtel pour accéder au questionnaire en ligne. Celui-ci ne pouvait être rempli qu'une seule fois par chaque utilisateur (blocage des tentatives d'accès multiples à travers les identifiants IP des utilisateurs).

Chaque questionnaire comportait entre 25 et 30 questions, dont certaines étaient identiques pour les deux enquêtes. Parmi les thématiques abordées figuraient : la fréquentation du quartier (à quel rythme et pourquoi), le sentiment de sécurité dans le quartier (de nuit, de jour, avant/après les caméras), la perception du système de caméras et de ses effets, ainsi que l'appréciation d'autres mesures sécuritaires. Des filtres appliqués au questionnaire rendaient la navigation pour les utilisateurs logique et cohérente ; certaines questions n'apparaissaient qu'à la condition que d'autres soient préalablement remplies.

\section{Connaissance populaire vis-à-vis des caméras aux Pâquis}

Pour que la vidéosurveillance puisse territorialiser symboliquement des espaces filmés, il est impératif que les usagers de ces espaces connaissent l'existence des caméras. Par conséquent, avant d'étudier l'acceptabilité de la vidéosurveillance aux Pâquis ainsi que ses effets en matière de sécurité subjective, d'image et de fréquentation du quartier, il est important de saisir dans quelle mesure les personnes interrogées avaient véritablement connaissance de la présence de caméras.

En 2015, 63\% des répondants affirmaient avoir connaissance des caméras aux Pâquis (Fig. 2). Ce taux était quasi identique une année plus tard $(65 \%)$. En ne considérant que les participants habitant dans la zone pilote, ce taux atteignait même $83 \%$ en 2015 et $84 \%$ en 2016. Dans les zones de contrôle, environ la moitié $(52 \%)$ des répondants disait connaître l'existence de la vidéosurveillance. Ce niveau de connaissances est sensiblement plus élevé que dans d'autres cas étudiés (Honess et Charman, 1992; Ditton, 2000; Klauser, 2006). En l'occurrence, les personnes interrogées disent en grande partie avoir appris l'existence des caméras à travers les médias ( $80 \%$ des répondants en 2015), alors que seulement $25 \%$ des répondants en ont pris connaissance en voyant les caméras dans la rue (Fig. 2). D'un diamètre de moins de trente centimètres et situées à plusieurs mètres du sol les caméras sont en effet peu visibles. C'est donc principalement le fort intérêt médiatique au moment de leur installation qui 


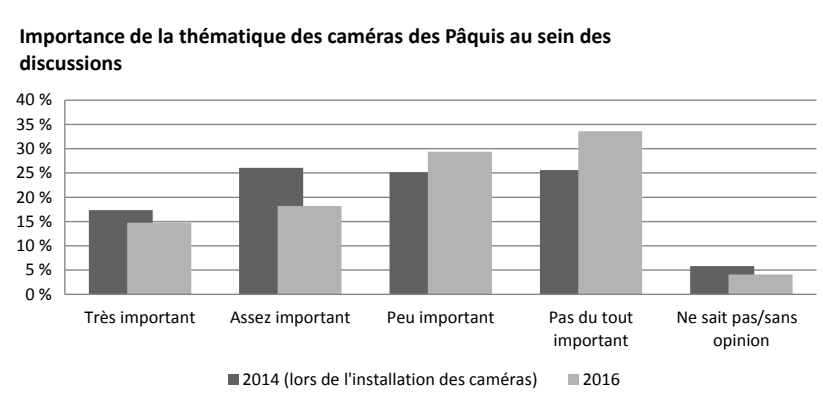

Figure 3. Perception de l'importance des caméras des Pâquis au sein des discussions des répondants (avec leurs amis/voisins/conjoint/famille) des zones pilote, tampon et de contrôle (Source questionnaire 2016, $N=652$ ).

a donné à la vidéosurveillance une très forte présence symbolique.

Or, le haut degré de conscience des répondants concernant l'existence des caméras ne signifie pas forcément qu'ils les considèrent de facto comme étant un enjeu important dans leur quotidien. En 2015, respectivement 17 et $26 \%$ des répondants considéraient les caméras comme étant un sujet "très important" ou "assez important" au sein de leurs discussions. En 2016, ces taux étaient de 15 et $18 \%$ (Fig. 3). La part des répondants considérant la thématique des caméras comme étant "peu" ou "pas du tout importante" tend, quant à elle, à augmenter. Il semble que la vidéosurveillance ait perdu de l'importance au sein des discussions des habitants et usagers du quartier.

Dans la continuité du graphique précédent, nous pouvons également souligner la forte majorité de répondants estimant que les gens ont tendance à oublier la présence des caméras dans leur quotidien (79\% en 2016), confirmant encore un effet d'oubli et d'accoutumance vis-à-vis du dispositif. Avec le temps, la population tend à être moins attentive à la vidéosurveillance même si l'existence des caméras est a priori bien connue. Cette dynamique souligne la mise à distance mentale, précédemment présentée, qui résulte du caractère spatialement et socialement distant de cette forme de télérégulation (Guéniat et Klauser, 2013).

Par ailleurs, il est intéressant de noter que parallèlement à cette relégation graduelle des caméras à l'arrière-plan, les craintes initiales de la population quant au dispositif se sont, elles, également dissipées. Par exemple, le nombre de répondants craignant que les images enregistrées par les caméras ne soient vues par d'autres que la police (c'est-à-dire le risque de fuites d'images) est passé de $37 \%$ en 2015 à $25 \%$ en 2016. Certes, cette crainte n'a jamais été dominante parmi les personnes interrogées, même si elle n'était pas non-plus négligeable. En plus, la population approuve largement la surveillance étatique comme étant légitime et peu problématique : deux-tiers des répondents estiment qu'il est légitime que l'Etat filme l'espace public, et trois cinquièmes considèrent que les caméras ne sont pas une atteinte à leur sphère

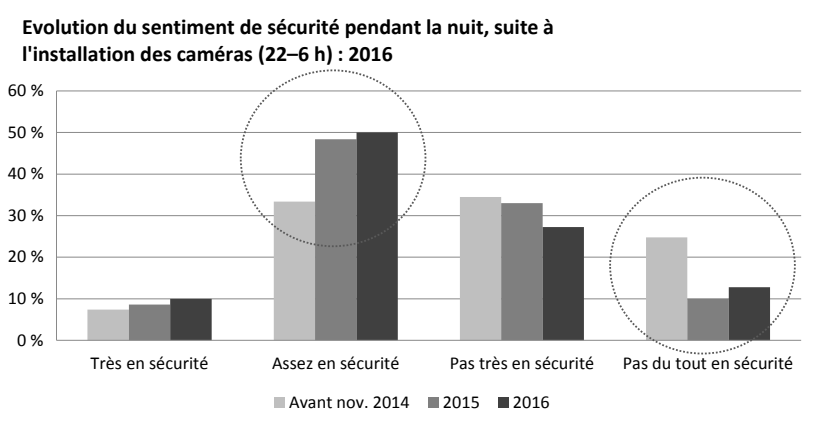

Figure 4. Evolution du sentiment de sécurité entre 22 et $6 \mathrm{~h}$ des répondants des zones pilote, tampon et de contrôle. Comparaison entre la situation d'avant l'installation des caméras (avant novembre 2014), la situation en 2015 et celle de 2016 (Source : questionnaire 2015, $N=815 / 2016, N=578)$.

privée (résultats quasi identiques pour 2015 et 2016). Toutefois, cette atténuation des craintes spécifiques vis-à-vis du projet des Pâquis permet de souligner le fait qu'en l'absence d'échos médiatiques au sujet des caméras, fussent-ils positifs ou négatifs, la population s'habitue vite à la vidéosurveillance, au point de l'oublier. Le système de caméras perd ainsi sa capacité à faire débat et se fond progressivement au sein du paysage urbain quotidien, tout en voyant son pouvoir de territorialisation symbolique diminuer.

\section{Evolution du sentiment de sécurité}

Si les caméras perdent graduellement de leur pouvoir symbolique, qu'en est-il de leurs effets sur le sentiment de sécurité des usagers des espaces filmés ? En répondant à cette question, nos deux enquêtes mettent d'abord en évidence un grand contraste entre le sentiment de sécurité de jour et de nuit. Dans l'enquête 2015, 89 \% des répondants affirmaient se sentir en sécurité aux Pâquis pendant la journée, alors que ce taux baissait à $57 \%$ pendant la nuit (Fig. 4). Si l'on compare ces chiffres avec le souvenir des répondants, à ce moment, de leur sentiment de sécurité avant l'installation des caméras, on voit que la vidéosurveillance a eu un effet rassurant dès sa mise en service, notamment pendant la nuit (Fig. 4). $48 \%$ des répondants en 2015 se disaient "assez en sécurité" pour la situation actuelle, alors que pour la situation d'avant ce chiffre était de $33 \%$. Inversement, le taux de répondants ne s'estimant "pas du tout en sécurité" chutait de 25 à $10 \%$ dès l'installation des caméras (pendant la nuit).

Avec l'enquête 2016, l'image concernant cet effet rassurant des caméras se complexifie. On constate toujours une légère amélioration du sentiment de sécurité nocturne par rapport à l'année précédente, et suite à l'installation des caméras, mais on note aussi une recrudescence des personnes qui ne se sentent "pas du tout en sécurité" (13\%). Parallèlement au recul de la médiatisation des caméras, la courbe initialement positive à l'égard du sentiment de sécurité des 
gens s'est ainsi étiolée et même légèrement renversée après deux ans, restant pourtant plus positive qu'avant les caméras. Il faudrait poursuivre cette étude avec une troisième année d'évaluation afin de savoir comment cette tendance évoluerait à plus long terme.

En outre, nous avons intégré dans l'enquête 2016 une série d'énoncés pouvant être approuvés/refusés par les répondants. Ces énoncés portent notamment sur trois catégories de bénéficiaires potentiels de la vidéosurveillance du point de vue de leur sentiment de sécurité : les personnes âgées, les familles avec enfants et les écoliers. Parmi les participants, les opinions à ce sujet se sont consensuelles. Respectivement, 43, 43 et $42 \%$ des répondants ressentent un effet rassurant des caméras pour ces catégories de population, avec des pourcentages quasi identiques pour les personnes en désaccord avec cet avis (36, 37 et 37 \%). Là encore, l'appréciation du pouvoir symbolique des caméras un an après leur installation est plus mitigée que ne le laissait penser l'évolution positive initiale du sentiment de sécurité nocturne. Tout comme les oppositions et les craintes, les espoirs liés à la vidéosurveillance se sont dissipés avec le temps.

À ce résultat peut être ajouté un autre graphique montrant que la population n'a pas observé de changements en matière d'évolution de la criminalité aux Pâquis (Fig. 5). A noter que ne sont pris en compte dans ce graphique que les répondants qui fréquentent les Pâquis au moins deux fois par mois. Nous considérons en effet qu'il faut être domicilié ou se rendre régulièrement dans le quartier pour porter un regard sur d'éventuels changements en matière de criminalité. Par ailleurs, les tendances du graphique sont identiques lorsque l'on ne considère que les répondants domiciliés dans la zone équipée de caméras.

Clairement, les répondants n'observent pas d'effets de la vidéosurveillance sur les problèmes de bruit, de trafic de stupéfiants ou sur les incivilités. Ce constat est moins marqué pour les actes de violence/bagarres ou vols dans la rue, mais la perception populaire de l'évolution de ces types de délits reste mitigée (31 et $30 \%$ pensent que les caméras ont diminué les actes de violence et les vols respectivement, et 35 et $28 \%$ ne partagent pas cet avis). Notons aussi que les taux de "Ne sait pas/Sans opinion" sont très importants pour cette question, notamment s'agissant de l'évolution des violences et des vols. Cela souligne les difficultés de la population à juger concrètement des vertus et des bénéfices des caméras. Les effets de la vidéosurveillance restent distants et largement méconnus.

En outre, la vidéosurveillance des Pâquis reste distante également du point de vue de son fonctionnement. Invités à se prononcer sur l'importance de certaines propositions au sein d'une liste de mesures visant à renforcer la sécurité des Pâquis, les répondants préfèrent celles qui portent sur la lutte contre le commerce de stupéfiants ainsi que celles qui visent à renforcer la présence policière sur le terrain. En effet, plus de $80 \%$ des répondants souhaitent une augmentation des patrouilles de police (Fig. 6). Les répondants se sont égale-

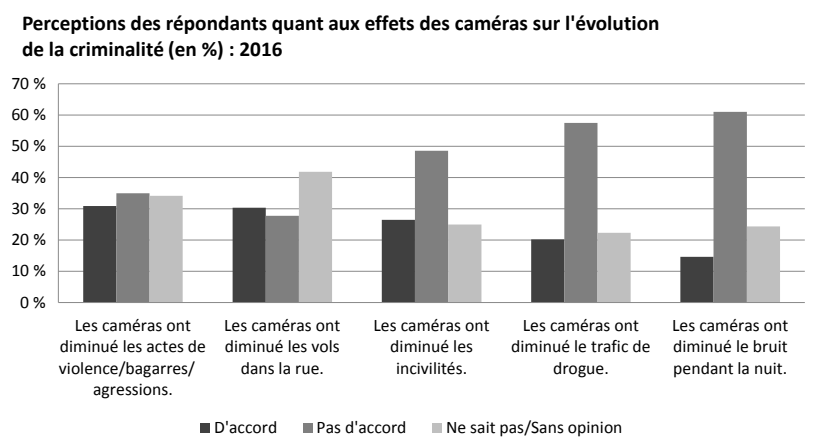

Figure 5. Perception des répondants concernant les effets des caméras sur différents types de criminalité (Source : questionnaire 2016, $N=568-572$ ).

ment montrés sensibles aux questions d'aménagements urbains (éclairage public ou aménagement des espaces publics) ainsi qu'aux mesures visant à renforcer la vie de quartier et les liens sociaux (événements de quartier, associations d'habitants, présence d'espaces sociaux, etc.). Les mesures visant à conserver ou à étendre le système de caméras à d'autres quartiers n'apparaissent qu'après ces premiers choix.

Ce résultat confirme qu'en cas de libre choix, la population préfère des mesures humaines plutôt que technologiques. Autrement dit, on observe une préférence marquée pour des mesures qui créent de la proximité plutôt que de la distance. A ce sujet, notons aussi que les répondants ne se sont pas majoritairement prononcés contre le caractère divertissant et festif des Pâquis (y compris la scène de la prostitution). A l'inverse, ils refusent les propositions suggérant de diminuer ou de fermer les établissements nocturnes ainsi que les commerces ouverts $24 / 24 \mathrm{~h}$. Il ne s'agit pas, dès lors, de changer le caractère animé et nocturne des Pâquis. La vidéosurveillance, à son tour, ne gêne pas, mais n'est pas activement plébiscitée. En somme, cela invite à une réflexion plus large concernant la vocation et le fonctionnement même de l'espace public comme espace de mixité sociale et de contrôle mutuel se faisant par le regard réciproque des personnes coprésentes.

\section{Modifications des pratiques spatiales dans le quartier}

Le dernier aspect de notre analyse concerne la perception populaire des effets de la vidéosurveillance sur l'image des Pâquis ainsi que sur sa fréquentation. Est-ce que la vidéosurveillance a contribué à reterritorialiser le quartier dans sa perception et fréquentation au-delà de l'aspect sécuritaire?

L'impact de la vidéosurveillance sur le dynamisme social et commercial d'un quartier reste difficile à établir, car les caméras s'intègrent généralement dans un ensemble de mesures plus vastes, et très peu d'études traitent de ces questions empiriquement. Les quelques recherches qui évoquent ces aspects soulignent souvent les motivations commerciales 


\section{Opinions des répondants quant à des propositions liées à la sécurité dans le quartier des Pâquis: 2015}

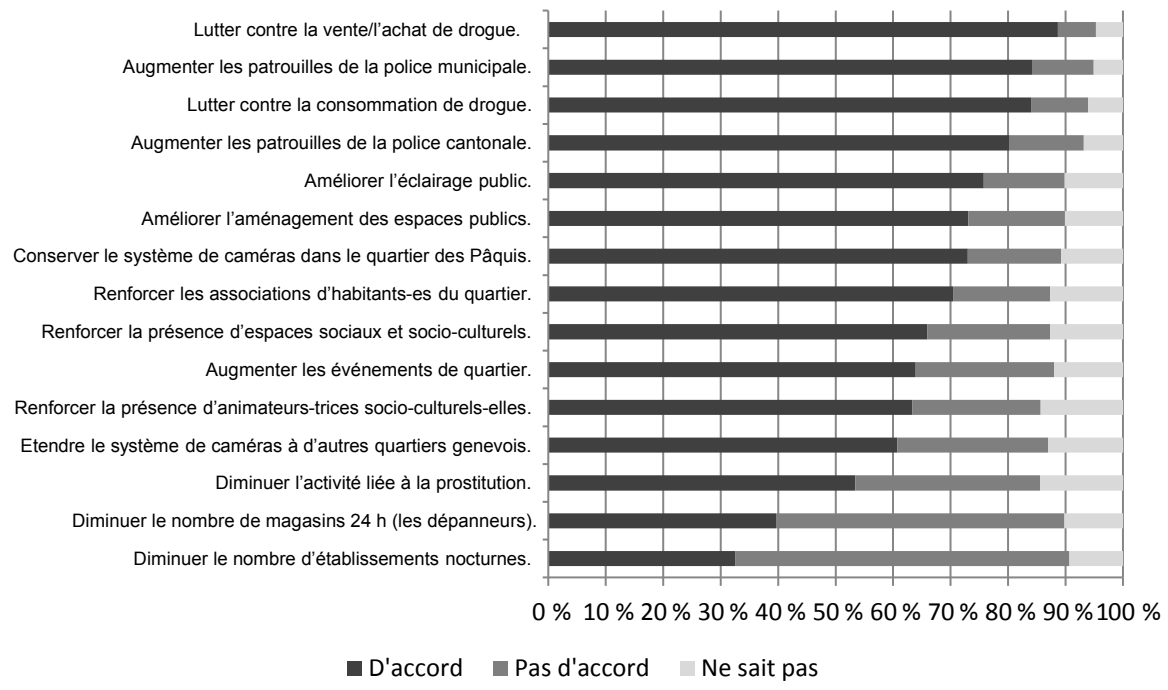

Figure 6. L'opinion des répondants des zones pilote, tampon et de contrôle quant à l'amélioration de la sécurité et de la qualité de vie au quartier des Pâquis (Source : questionnaire 2015, $N=880-911$ ).

sous-jacentes aux projets de vidéosurveillance. Ces enjeux sont notamment abordés en lien à la gentrification et à la commercialisation des centre villes (Coleman et Sim, 2000; Martinais et Bétin, 2004), tout en démontrant qu'en fin de compte, les caméras n'engendrent pas de grands bouleversements concrets (Heilmann, 2003; Klauser, 2007).

Dans cette optique, la question des effets de la vidéosurveillance sur l'image d'un quartier est cruciale, car elle touche non seulement à la réputation du quartier, mais aussi à la perception de toute une série d'autres éléments, tels que l'environnement bâti, son ambiance, et les activités qui y prennent place. En l'occurrence, notons d'abord que $44 \%$ des répondants de notre enquête estimaient en 2016 que la présence des caméras aux Pâquis avait contribué à améliorer l'image du quartier (Fig. 7). En même temps, $35 \%$ des répondants ne voyaient pas d'incidence des caméras à cet égard et $13 \%$ estimaient que les caméras avaient même un effet négatif, car stigmatisant. Signalons en passant l'absence de différences significatives entre les opinions des habitants des Pâquis et ceux des zones test. Dans l'ensemble, l'avis populaire quant aux effets de la vidéosurveillance sur la réputation des Pâquis est partagé.

Si l'on passe maintenant à la question de la fréquentation, l'absence presque complète de changements d'usages observés ou imaginés aux Pâquis est frappante : seulement un cinquième des répondants remarque une augmentation générale de la fréquentation des Pâquis depuis l'installation des caméras. Une même proportion de personnes observe que la fréquentation des touristes ou encore des noctambules a augmenté dans le quartier. A ce sujet, une comparaison des
Perception des répondants quant aux effets de la vidéosurveillance sur l'image du quartier des Pâquis: 2016

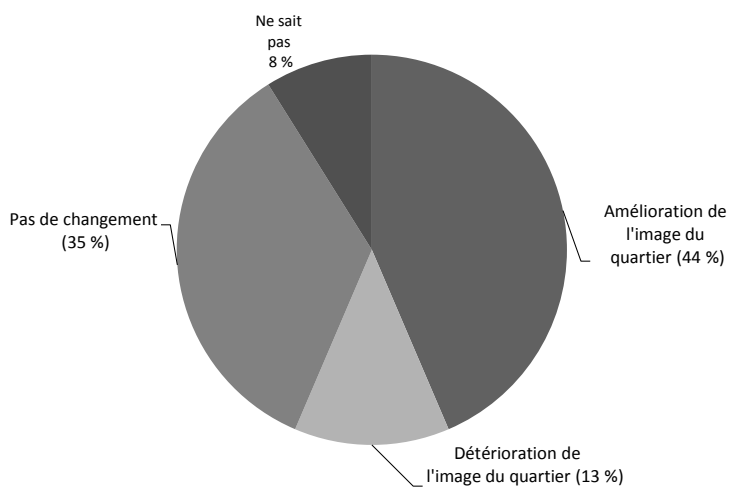

Figure 7. Perception de l'image du quartier par les répondants des zones pilote, tampon et de contrôle quant à l'image du quartier (Source : questionnaire 2016, $N=1029$ ).

réponses obtenues selon le lieu d'habitation des répondants s'avère intéressante (Fig. 8a et b).

Premièrement, notons les pourcentages importants de personnes qui, elles-mêmes, ne fréquentent pas plus le quartier depuis l'installation des caméras. Cette réponse est logique pour les personnes habitant aux Pâquis et aux alentours; on verrait mal pourquoi les caméras leur feraient changer de parcours. Mais concernant les habitants des autres quartiers cette question démontre que l'amélioration du sentiment de sécurité constatée auparavant ne s'est guère traduite en changement de pratiques concrètes. 
Perception des habitants des Pâquis (zone pilote + tampon) quant à la

(a)

fréquentation du quartier depuis l'installation des caméras en 2016.

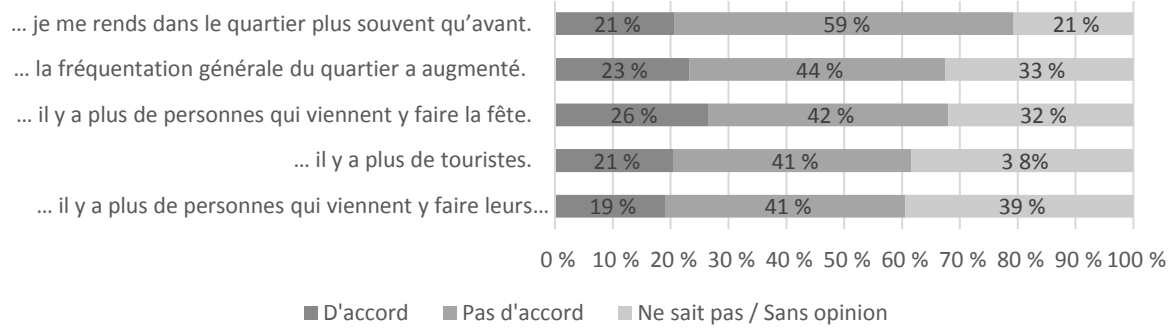

Perception des habitants d'autres quartiers (zones de contrôle) quant à la fréquentation du quartier depuis l'installation des caméras en 2016.

(b)

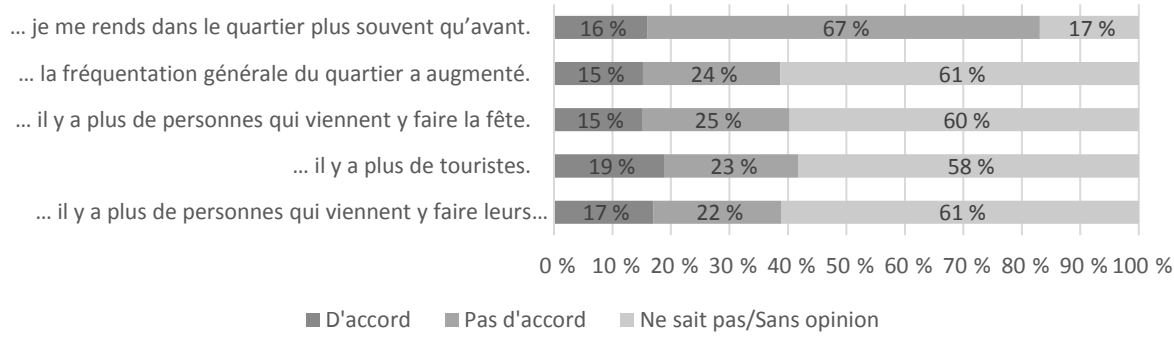

Figure 8. Perception des répondants des zones pilote et tampon (en haut) et des zones de contrôle (en bas) quant aux changements d'usages du quartier après l'installation des caméras (Source : questionnaire 2016, $N$ réponses par questions $=555-574$ ).

Deuxièmement, les pourcentages élevés de réponses du type "Ne sait pas" ou "Sans opinion", surtout pour les habitants des zones test, mettent en évidence le haut niveau d'ignorance vis-à-vis d'éventuels effets spécifiques des caméras. Tout comme le suggéraient les résultats précédents sur l'évolution du sentiment de sécurité, nous retrouvons un certain optimisme de base quant aux effets diffus des caméras sur l'image du quartier, alors que les répondants sont plus hésitants quand il s'agit d'identifier des bénéfices ou des bénéficiaires spécifiques. En somme, retenons que l'installation des caméras ne semble pas avoir reterritorialisé le quartier du point de vue de son affluence.

Malgré cela, la moitié des répondants (soit exactement $50 \%$ en 2016) s'est exprimée non seulement en faveur d'un maintien des caméras, mais aussi d'une extension du système à d'autres quartiers genevois. Un quart (25\%) s'est prononcé uniquement pour le maintien des caméras et seulement $15 \%$ des répondants souhaiteraient voir disparaître les caméras des Pâquis. Même si les répondants ne se font pas d'illusions quant aux impacts des caméras, et même s'ils n'observent pas de changements concrets à l'échelle du quartier, les caméras restent néanmoins bien accueillies par la population.

\section{Conclusions}

Notre analyse invite à relativiser le pouvoir de territorialisation symbolique inhérent à la vidéosurveillance. Ceci pour trois raisons principales. Premièrement, les résultats provenant des deux enquêtes successives confirment que l'efficacité de la vidéosurveillance en tant qu'instrument de territorialisation symbolique à distance dépend fondamentalement du facteur temps. Même si une large part de la population interrogée a connaissance de l'existence des caméras aux Pâquis, l'intérêt que présentaient les caméras en tout début de projet (à leur installation) a graduellement diminué entre 2015 et 2016. Le sujet occupait bien les conversations au départ, mais n'était déjà plus d'actualité un an et demi après. Ceci malgré les échos médiatiques importants lors de l'annonce de la mise en service des caméras. Les caméras ont ainsi été successivement reléguées à l'arrière-plan des préoccupations quotidiennes des habitants et usagers du quartier.

Deuxièmement, s'agissant des effets perçus de la vidéosurveillance, notre étude met en évidence le contraste entre l'accueil favorable et optimiste du dispositif - exprimé sous forme d'un renforcement du sentiment de sécurité et d'une perception positive des caméras en matière d'image du quartier - et l'impossibilité pour les répondants d'identifier des bénéfices spécifiques pour le quartier. La vidéosurveillance, dans ses effets, reste relativement diffuse et abstraite. L'idée générale que s'en fait la population est positive, mais le 
manque d'informations spécifiques et l'absence de changements concrets et observables dans le quartier ne permettent pas une appropriation citoyenne complète des caméras. Il n'est guère surprenant, sur cette base, qu'une police de proximité qui maintiendrait un contact proche avec la population, ou encore des mesures renforçant les liens sociaux à l'échelle $\mathrm{du}$ quartier (associations, évènements, etc.) soient perçues par la population comme étant plus utiles et rassurantes que la surveillance par caméra vidéo.

Troisièmement, les caméras ont certes eu des effets positifs initiaux concernant le sentiment de sécurité nocturne aux Pâquis, mais celui-ci ne se traduit pas en une fréquentation accrue du quartier. Or, le pouvoir symbolique de la vidéosurveillance n'est que virtuel tant que les usagers des espaces surveillés ne l'actualisent pas. Le pouvoir de territorialisation symbolique inhérent à la vidéosurveillance n'a ainsi pas réalisé son plein potentiel, en n'affectant pas véritablement la vie sociale du quartier.

Ces éléments viennent corroborer les conclusions d'autres études, qui démontrent que la distance, spatiale et sociale, inhérente à la vidéosurveillance se traduit également en une distance mentale, caractérisant le rapport des surveillés aux caméras. La majorité de la population n'est pas gênée par la présence des caméras et reste même plutôt favorable à leur installation, mais l'intérêt porté au dispositif disparaît rapidement. Ceci notamment parce que les caméras manquent d'éléments humains de proximité, qui favorisaient une appropriation populaire plus complète et plus durable du dispositif. En l'occurrence, cette distance mentale a également contribué à une certaine banalisation de la vidéosurveillance aux yeux des personnes interrogées. Les caméras sont devenues ordinaires, perçues comme un système pouvant potentiellement apporter des bénéfices, bien que nul ne sache vraiment pour qui et comment. Dès lors, les comportements des habitants et usagers ne changent pas et rares sont ceux qui s'interrogent quant à des éventuels problèmes soulevés ou des effets négatifs.

Ainsi, le fait de déléguer la régulation d'un espace à des systèmes et à des individus qui en sont séparés physiquement risque en effet de nuire à l'intégration de la société civile dans des pratiques sécuritaires de proximité (Ruegg et al., 2007). Ceci peut s'avérer problématique dans la mesure où la délégation de la surveillance à des acteurs à distants éloigne l'Etat et le citoyen du lieu de débat, soit de la situation de coprésence dans l'espace public; précisément là où les incivilités devraient être régulées. Ceci soutient l'argument que la présence policière visible au sein de l'espace public doit rester la mesure prioritaire en matière de sécurité et que les caméras ne représentent ainsi rien d'autre qu'un outil complémentaire. Cette conclusion n'est pas sans lien avec la crainte souvent exprimée, dans le cadre de projets de caméras, de voir la police remplacée par des caméras. Pourtant, dans le cas genevois, les statistiques policières indiquent une présence stable des patrouilles sur le terrain (Kaenzig et Klauser, 2016).
Pour compléter l'image mitigée résultant de la vidéosurveillance comme instrument de territorialisation symbolique des espaces observés à distance, deux choses seraient à entreprendre. Premièrement, il faudrait continuer à suivre l'évolution de la perception populaire des caméras aux Pâquis et de ses effets. Une attention particulière devrait porter sur la manière dont la distance mentale saisie et décrite dans notre analyse continue d'évoluer dans le temps, notamment sous l'effet d'éventuels succès du dispositif (crimes élucidés). Deuxièmement, afin de générer une image plus complète du pouvoir symbolique des caméras, les effets préventifs du dispositif quant à l'évolution des statistiques de la criminalité dans le quartier seraient à analyser. Le présent article est donc volontairement limité à des questions de sécurité subjective, mais cette analyse sera en effet complétée par une publication future consacrée aux effets des caméras en termes de criminalité, soit la dimension préventive.

\section{Données disponibles}

Les données quantitatives sous-jacentes à ce document ne sont pas publiquement disponibles. Pour plus d'information, veuillez contacter les auteurs.

Intérêts concurrents. Les auteurs déclarent qu'ils n'ont acun conflit d'intérêts.

Remerciements. Les auteurs remercient les personnes suivantes pour leurs apports multiples et variés aux différentes étapes de cette étude : Noémie Béguelin Caudoux, Romaric Thiévent, Sarah Widmer, Geoffrey Klein, Bernard Taschini, André Faggioni, Didier Froideveaux, ainsi que les membres du groupe d'accompagnement mis en place pour le projet.

Edited by: Myriam Houssay-Holzschuch

Reviewed by: two anonymous referees

\section{Références}

Akrich, M. et Méadel, C.: Anthropologie de la télésurveillance en milieu privé, École des Mines, Centre de Sociologie de l'Innovation, Paris, 1999.

Armitage, R.: To CCTV or not to CCTV. A Review of Current Research into the Effectiveness of CCTV Systems in Reducing Crime, National Association for the Care and Resettlement of Offenders, London, 2002.

Armstrong, G. et Giulianotti, R.: From another angle: Police surveillance and football supporters, in: Surveillance, Closed Circuit Television and Social Control, édité par: Norris, C., Moran, J. et Armstrong, G., Ashgate, Aldershot, 113-135, 1998.

Barbichon, G.: Espaces partagés: Variation et variété des cultures, Espaces et sociétés, 62-63, 107-133, 1990. 
Bowers, K. J. et Johnson, S. D.: Measuring the geographical displacement and diffusion of benefit effects of crime prevention activity, Journal of Quantitative Criminology, 19, 275-301, 2003.

Brown, B.: CCTV in Town Centres: Three Case Studies, Home Office, London, 1995.

Carli, V.: La vidéosurveillance est-elle un outil de sécurité et de gestion efficace pour lutter contre la criminalité, faire baisser le taux de criminalité et renforcer le sentiment de sécurité?, Rapport de recherche, Centre international pour la prévention de la criminalité, Montréal, 2009.

Coleman, R. et Sim, J.: You'll never walk alone: CCTV surveillance, order and neo-liberal rule in Liverpool city centre, British Journal of Sociology, 51, 623-639, 2000.

Département de la sécurité et de l'économie du Canton de Genève: La vidéoprotection devient réalité aux Pâquis, Communiqué de presse, 2014.

Ditton, J.: Public support for town centre CCTV schemes: Myth or reality?, in: Surveillance, Closed Circuit Television and Social Control, édité par: Norris, C., Moran, J. et Armstrong, G., Ashgate, Aldershot, 221-228, 1998.

Ditton, J.: Crime and the city: public attitudes towards Open-street CCTV in Glasgow, Brit. J. Criminol, 40, 692-709, 2000.

Ditton, J. et Short, E.: Yes, it works, no, it doesn't: Comparing the effects of open-street CCTV in two adjacent Scottish town centres, Crime Prevention Studies, 10, 371-403, 1999.

Fonteneau, M.: Vidéosurveillance et espaces publics: État des lieux des évaluations menées en France et à l'étranger, Institut d'Aménagement et d'Urbanisme, Paris, 2008.

Fyfe, N. R. et Bannister, J.: THE EYES UPON THE STREET, Images of the Street: Planning, Identity, and Control in Public Space, Home Office, London, 1998.

Gill, M. et Spriggs, A.: Assessing the impact of CCTV, Home Office, London, 2005.

Guéniat, O. et Klauser, F.: La vidéosurveillance: Quelle efficacité, l'Hebdo, 86-88, 2013.

Heilmann, E.: La vidéosurveillance, une réponse efficace à la criminalité? Criminologie, 36, 89-102, 2003.

Heilmann, E., Douillet, A. C., Dallaserra, J., Melchior, P. et Germain, S.: Vidéo-surveillance ou vidéo-protection, Le Muscadier, Paris, 2012.

Home Office: National CCTV Strategy, Rapport de recherche, Home Office, London, 2007.

Honess, T. et Charman, E.: Closed Circuit Television in Public Places: Its Accebtability and Perceived Effectiveness, Home Office, London, 1992.

Jeudy, H.-P.: Norme et transgression dans l'espace public, Espaces et Sociétés, 62-63, 83-91, 1990.

Kaenzig, R. et Klauser, F.: Evaluation de la vidéoprotection dans le quartier des Pâquis (2014-2016), Rapport de Recherche, Institut de Géographie, Université de Neuchâtel, 2016.

Klauser, F.: La vidéosurveillance de l'espace public: Nouvelle forme d'appropriation spatiale?, in: NTIC et territoires. Enjeux territoriaux des nouvelles technologies de l'information et de la communication, édité par: Vodoz, L., Presses Polytechniques et Universitaires Romandes, Lausanne, 87-94, 2001.

Klauser, F.: Die Videoüberwachung öffentlicher Räume: zur Ambivalenz eines Instruments sozialer Kontrolle, Campus Verlag, Frankfurt am Main, 2006.
Klauser, F.: Disturbances in the experience of the city through our senses: CCTV and the development of an unreal parallel world, Senses and Society, 2, 173-187, 2007.

Klocke, G.: Das Hintertürchen des Nichtwissens, Cilip, 2, available at: https://www.cilip.de/2001/08/09/das-hintertuerchen-desnichtwissens (dernier accès: 18 janvier 2017), 2001.

Korosec-Serfaty, P.: Le public et ses domaines, Contribution de l'histoire des mentalités à l'étude de la sociabilité publique et privée, Espaces et sociétés, 62-63, 29-59, 1990.

Koskela, H.: Fear, Contraol and Space. Geographies of Gender, Fear of Violence and Video Surveillance, Publications of the Department of Geography, University of Helsinki, Helsinki, 1999.

Marx, G. T.: The new surveillance, National Forum, 71, 32-36, 1991.

Mach, P. et Feuz, A.: Temps Présent: Les petites soeurs de big brother, Television Suisse Romande la première, available at: http://www.rts.ch/emissions/temps-present/societe-moeurs/ 1324449-les-petites-soeurs-de-big-brother.html (dernier accès: 18 janvier 2017), 2007.

Martinais, E. et Bétin, C.: Social aspects of CCTV in France: The case of the city centre of Lyons, Surveillance and Society, 2, 361375, 2004.

Mendicino, F.: Poignardé au thorax en plein cœur des Pâquis, Tribune de Genève, available at: http://www.tdg.ch/ geneve/actu-genevoise/poignarde-thorax-plein-cur-pquis/story/ 13497303 (dernier accès: 18 janvier 2017), 2015.

Norris, C. et Armstrong, G.: Introduction: Power and vision, in: Surveillance, Closed Circuit Television and Social Control, édité par: Norris, C., Moran, J. et Armstrong, G., Ashgate, Aldershot, 3-18, 1998.

November, V., Ruegg, J. et Klauser, F.: Vidéosurveillance: mécanismes de régulation dans l'espace à usage public, Rapport de recherché, COST, Université de Genève, Genève, 2003.

Pellegrino, P., Lambert, C. et Jacot, F.: Espace public et figures du lien social, Espaces et Sociétés, 62-63, 11-27, 1990.

Raffestin, C.: Pour une géographie du pouvoir, Litec, Paris, 1980.

République et Canton de Genève: Politique commune en matière de lutte contre la criminalité 2014-2016, Convention signée le 17 septembre 2014, Genève, 2014.

Ruegg, J., Flückiger, A., November, V. et Klauser, F.: Vidéosurveillance et risques dans l'espace à usage public. Représentations des risques, régulation sociale et liberté de mouvement, Rapport de recherche, Fonds National Suisse, Université de Fribourg, Fribourg, 2006.

Ruegg, J., Klauser, F. et November, V.: Du citoyen et de la civilité: Réflexions à partir de l'exemple de la vidéosurveillance, Lien Social et Politique, 57, 127-39, 2007.

Sivarajasingam, V., Shepherd, J. P. et Matthews, K.: Effect of urban closed circuit television on assault injury and violence detection, Injury Prevention, 9, 312-316, 2003.

Skinns, D.: Crime reduction, diffusion and displacement: Evaluating the effectiveness of CCTV, in: Surveillance, Closed Circuit Television and Social Control, édité par: Norris, C., Moran, J. et Armstrong, G., Ashgate, Aldershot, 175-188, 1998.

Smith, G. J. D.: Opening the Black Box. The Work of Watching, Routledge, London, 2015.

Taylor, E.: Surveillance Schools: Security, Discipline and Control in Contemporary Education, Palgrave Macmillan, Basingstoke, 2013. 
Tilley, N.: Evaluating the effectiveness of CCTV schemes, in: Surveillance, Closed Circuit Television and Social Control, édité par: Norris, C., Moran, J. et Armstrong, G., Ashgate, Aldershot, 139-153, 1998.

Waples, S., Gill, M. et Fisher, P.: Does CCTV displace crime?, Criminology and Criminal Justice, 9, 207-224, 2009.

Welsh, B. C. et Farrington, D. P.: Crime Prevention Effects of Closed Circuit Television: A Systematic Review, Home Office, London, 2002.

Williams, K., Johnstone, C. et Goodwin, M.: CCTV surveillance in urban Britain: Beyond the rhetoric of crime prevention, in: Landscapes of Defence, édité par: Gold, J. et Revill, G., Prentice Hall, Harlow, 168-187, 2000.
Wünsch, A.: Videoüberwachung: Psychologische Aspekte, Sicherheitsforum, 5, 137-141, 2001.

Zehnder, M.: Kameraüberwachung als Präventionsinstrument im öffentlichen urbanen Raum, Evaluation für den Bahnhofplatz der Stadt Luzern, Rapport de recherche, Université de Bâle, Bâle, 2011.

Zurawski, N.: Raum - Weltbild - Kontrolle: Raumvorstellungen als Grundlage gesellschaftlicher Ordnung und ihrer Überwachung, Budrich UniPress, Opladen, 2014. 